\title{
Anti-tuberculosis drug resistance pattern among pulmonary tuberculosis patients with or without HIV infection in Mwanza, Tanzania
}

\author{
NYAGOSYA RANGE ${ }^{*}$, HENRIK FRIIS ${ }^{2}$, SAID MFAUME ${ }^{1}$, PASCAL MAGNUSSEN ${ }^{3}$, JOHN \\ CHANGALUCHA $^{4}$, ANDREW KILALE ${ }^{1}$, APOLINARY MUGOMELA ${ }^{5}$ and AASE B. ANDERSEN ${ }^{6}$ \\ ${ }^{1}$ National Institute for Medical Research, Muhimbili Medical Research Centre, Dar es Salaam, Tanzania \\ ${ }^{2}$ Department of Human Nutrition, Faculty of Life Sciences, University of Copenhagen, Frederiksberg, Denmark \\ ${ }^{3}$ DBL-Institute for Health Research and Development, Faculty of Life Sciences, University of Copenhagen, \\ Frederiksberg, Denmark \\ ${ }^{4}$ National Institute for Medical Research, Mwanza Medical Research Centre, Mwanza, Tanzania \\ ${ }^{5}$ Zonal Tuberculosis Reference Laboratories, Bugando Medical Centre, Mwanza, Tanzania \\ ${ }^{6}$ Department of Infectious Diseases, University of Copenhagen, Rigshospitalet, Copenhagen, Denmark
}

\begin{abstract}
Anti-tuberculosis drug resistance is a major problem in tuberculosis (TB) control, particularly multi-drug resistance TB (MDR-TB). The objective of this study was to determine the prevalence of primary and acquired anti-TB drug resistance among newly diagnosed pulmonary TB (PTB) and relapse cases. Sputa were collected from newly diagnosed and relapse PTB patients. Drug susceptibility tests (DST) were performed on sputum culture positive isolates of Mycobacterium tuberculosis using resistance ratio method on four first-line anti-TB drugs: rifampicin, isoniazid, ethambutol and streptomycin. Demographic and anthropometric information was collected and HIV status was determined. Of the 523 culture positive isolates, DST results were available for 503 (96\%), 455 were new and 48 were relapse cases. Resistance to at least one of the four drugs was observed in $7.8 \%$ (39/503) of the isolates, $7.3 \%$ (33/455) were new and $12.5 \%$ (6/48) were from relapse cases. Mono resistance to isoniazid was higher in both among new 45.5\% (15/33) and relapse $50.0 \%$ (3/6) cases. Resistance to rifampicin and streptomycin alone was equal 4/33 (12.1\%) and only among new cases. Resistance to ethambutol alone was only one among new cases. Overall MDR-TB prevalence was $2.4 \%$ (12/503), nine were new and three were relapse cases. MDR-TB was 17.9\% (7/39) for rifampicin and isoniazid. Prevalence of HIV was $43.3 \%$ and was similar among new and relapse cases and not risk factor for drug resistance. Majority of PTB patients (52\%) had BMI below $18 \mathrm{~kg} / \mathrm{m}^{2}$. Those with BMI greater than $18 \mathrm{~kg} / \mathrm{m}^{2}$ were more likely to develop drug resistance than those with BMI below $18 \mathrm{~kg} / \mathrm{m}^{2}$ $(P=0.004)$. With the resurgence of TB and the high prevalence of HIV among TB patients, prevalence of drug resistance is still low both among new and relapses cases. Despite the current low drug resistance, there is a need for continuous monitoring of the resistance.
\end{abstract}

Keywords: Pulmonary tuberculosis, HIV, anti-tuberculosis, relapse, drug resistance, Tanzania

\section{Introduction}

Tuberculosis (TB) is one of the most serious infectious diseases world-wide. Among the 22 countries in the world with high TB burden (HBC), Tanzania ranks number 15 (WHO, 2011). AntiTB drug resistance is a major public health problem that threatens the success of directly observed therapy strategy (DOTS). Based on the World Health Organisation statistics, there were 500,000 cases of multi-drug resistance TB (MDR-TB) worldwide in 2006 which represents about 5\% of all cases of TB (WHO, 2008). The report showed that there was connection between MDR-TB and

\footnotetext{
* Correspondence: Nyagosya S. Range; E-mail: hrange08@gmail.com
} 
HIV. The connection was attributed to poor infection control measures rather than "host" factors. In Tanzania, according to the national anti-TB drug resistance surveillance done in 2007, the prevalence of MDR-TB was $1.1 \%$ and $3.9 \%$ among new and re-treatment cases, respectively (Chonde et al., 2010). This level of drug resistance in Tanzania is said to be among the lowest in the African countries with an estimated level of drug resistance.

Emergence of drug resistance in M. Tuberculosis has been associated with a variety of management factors such as (i) health provider and patient-related factors, including deficient TB control programmes resulting in inadequate administration of effective treatment; (ii) poor case holding, inadequate or irregular drug supply; (iii) poor patient adherence or non-adherence of patients to the prescribed drug therapy; (iv) the epidemic of HIV infection; (v) interruption of chemotherapy due to side effects; and (vi) use of anti-TB drugs for indications other than TB (Mahmoudi \& Iseman, 1993; Paramasvan \& Venkataraman, 2004). Essentially drug resistance arises in areas with poor TB control programmes and is said to be a man-made problem.

Despite the long existence of standard anti-TB regimen, low success rates continue to hamper TB control strategy, this result in increased anti-TB drug resistance, including MDR-TB and of recent extensively drug resistance TB (XDR-TB). The XDR-TB is an extremely serious, emerging threat to public health and TB control (Masjedi et al., 2008; WHO, 2011). Epidemiological studies to assess local resistance rates and the detection of MDR-TB are therefore crucial to optimize empirical drug therapy and to prevent the dissemination of resistant strains in the community. This study was therefore conducted to determine primary and acquired anti-TB drug resistance levels among newly diagnosed pulmonary TB (PTB) patients and relapse cases in Mwanza, Tanzania.

\section{Materials and Methods}

\section{Study population and design}

Newly diagnosed PTB and relapses patients, aged 15 years or above who were residents of Mwanza City or Magu district in Mwanza region of north-western Tanzania were consecutively recruited from five health facilities between August 2001 and July 2002. Recruitment centres were Sekou Toure Regional Hospital, Bugando Medical Centre, Butimba and Buzuruga Health Centres and Magu District Hospital.

The original study was a randomized, placebo-controlled trial, to assess the effects of daily supplementation with a multi-micronutrient and a zinc tablet throughout the eight months of short course TB treatment (Range et al., 2005). The current study is a prospective cross sectional study to determine the level of initial (primary) drug resistance among newly diagnosed PTB patients and acquired (secondary) drug resistance among relapse (previously treated) cases using original data.

\section{Methods}

Suspects of TB were confirmed through clinical examination, smear microscopy, culture and or chest x-ray. All identified patients were requested to submit early morning sputum specimen in a universal bottle for subsequent smear microscopy and culture at the Zonal TB Reference Laboratory (ZTRL) at Bugando Medical Centre (BMC). Sputum samples for culture were collected before initiation of TB treatment at the recruitment centres. Smear microscopy at the recruitment 
centres was done using Ziehl-Neelsen staining technique (Bwire et al., 1999) while at the ZTRL; Auramine $\mathrm{O}$ staining technique was used. Culture was done on Lowenstein Jensen (LJ) solid media (Githui et al., 1993). Cultured samples were incubated at $37^{\circ} \mathrm{C}$ for up to eight weeks for primary isolation of $M$. tuberculosis. The positive culture isolates were sent to the Central TB Reference Laboratory (CTRL) in Dar es Salaam, for anti-TB drug susceptibility test (DST).

Prior to starting treatment, demographic and medical history data and anthropometric (height, weight, triceps skin fold thickness and upper arm circumference) measurements were taken from all patients. A $10-\mathrm{ml}$ venous blood sample was collected from each patient and used to check for HIV status. The HIV status was determined using two rapid tests, Determine HIV 1/2 (Inverness Medical Innovations Inc., Delaware, USA) and Capillus HIV-1/HIV-2 (Trinity Biotech Plc., Wicklow, Ireland). If the tests were equivocal, HIV diagnosis was based on ELISA. Questionnaires were administered to elicit information on age, marital status and occupation (Range et al. 2007).

Drug sensitivity testing was performed at the CTRL. Testing was done to four of the firstline anti-TB drugs namely, rifampicin $(\mathrm{R})$, isoniazid $(\mathrm{H})$, ethambutol $(\mathrm{E})$ and streptomycin $(\mathrm{S})$ using resistance ratio method. Fresh isolates (not exceeding 14 days after growth) with enough growth (50 colonies or more) were processed for DST immediately. Those cultures with few colonies or exceeded 14 days after first growth were sub-cultured onto fresh media. Colonies were scooped (about 1mg) using Niclechrome wire loop. Colonies were emulsified into sterile distilled water into 5 -ml bijou bottle containing 6 glass beads ( $3 \mathrm{~mm}$ diameter). Thereafter the samples were homogenized by agitating using electrical shaker for 1 minute. A mixture was diluted 10x and 100x by adding $0.8 \mathrm{ml}$ into $0.2 \mathrm{ml}$ of a sample (tube 1), then transferred 0.5 of the $10 \mathrm{x}$ into $4.5 \mathrm{ml}$ distilled water (tube 2). The dilutions were inoculated in two slopes of drug free media and drugs containing media. The drug free slopes were used as control for testing viability of the test organisms and drugs containing media were used to detect susceptibility of the test organisms. After inoculation, samples were incubated at $37^{\circ} \mathrm{C}$ for up to four weeks. H37RV strain was used as control organism.

The resistance ratio method is derived from dividing minimum inhibitory concentration (MIC) of the test strain over that of control strain, H37RV; i. e. RR = MIC test/ MIC of control. For isoniazid = MIC of $0.2 \mathrm{ng} / \mathrm{Ml}$ or more was regarded as resistant, for rifampicin, streptomycin and ethambutol; RR of 2 or less = sensitive; RR of $4=$ resistance; RR of $8=$ highly resistance.

\section{Data analysis}

Data was entered in SPSS version 11.5 SPSS for Windows and was analyzed using STATA version 8.0 (StataCorp. Texas USA). Level of statistical significance was set at 5\%.

\section{Definitions}

A new case is defined as a patient who has never had treatment for TB before or has been on treatment for not more than four weeks. Relapse case is defined as a patient declared cured or treatment completed but who reports back to the health facility and is found to be acid-fast bacilli (AFB) positive. A resistant case is defined as any individual who was resistant to any one of the drug tested either as single drug or in a combination of two or more drugs. Primary drug resistance is the presence of resistant strains of $M . T B$ in a newly diagnosed patient who has never 
received $\mathrm{TB}$ drugs or has received them for less than one month of treatment. Acquired or secondary drug resistance is the resistance found in a patient who has previously received at least one month of TB therapy. Multi-drug resistant TB (MDR-TB) is defined as disease due to M. TB that is resistance to at least two first-line drugs; rifampicin and isoniazid (WHO, 2011).

\section{Ethical consideration}

This study received ethical approval from the Medical Research Coordinating Committee of the National Institute for Medical Research in Tanzania and the Danish National Committee on Biomedical Research Ethics. Patients were given information about the study and offered inclusion after informed consent. All confirmed PTB patients at the recruitment centres were started on TB treatment based on the National TB treatment guidelines.

\section{Results}

A total of 523 PTB patients had smear and culture results done. Forty PTB patients had initial smear negative results but were culture positive. Of these, $70 \%(28 / 40)$ were HIV positive. Out of the 523 culture positive results, 20 isolates were contaminated and hence sensitivity result was available for 503 isolates. Of these, 455 (90.5\%) were new PTB patients and 48 (9.5\%) were relapse cases. Mean (SD) age was 35.6 years (11.9) ranging from 15-85 years. More than half of the PTB patients (52\%) 260/503) had body mass index (BMI) below $18 \mathrm{~kg} / \mathrm{m}^{2}$. The overall HIV prevalence was $43.3 \%$ (Table 1 ).

The proportion of patients who were sensitive or resistant to one or more drugs did not differ by sex, age group, HIV status, intensity of sputum smear or culture and health facility $(P>0.05)$. However, those with body mass index (BMI) greater than $18 \mathrm{~kg} / \mathrm{m}^{2}$ were more likely to have resistant strains compared to those with BMI less than $18 \mathrm{~kg} / \mathrm{M}^{2}(64.1$ vs 35.9, $P=0.04)$ (Table 1). Of the 503 whose susceptibility result was available, 464 (92.2\%) were sensitive to all four firstline anti-tuberculosis drugs and only 39 (7.8\%) were resistant to either one or more of the drugs tested (Table 2).

The primary or initial TB drug resistance was $84.6 \%$ (33/39). Of the 33 primary resistance strains, $28(84.85 \%)$ were from new smear positive and $5(15.15 \%)$ were from new smear negative PTB patients. Nine had MDR-TB, the remaining had mono resistance. The most common mono resistant was with isoniazid 15/33 (45.5\%) (Table 2). The acquired or secondary drug resistance was $16 / 39$ (15.38\%). Of these, three had MDR-TB and other three were mono-resistant to isoniazid.

Resistance to all four drugs, rifampicin, isoniazid, ethambutol and streptomycin (RHES) was observed in 3 out of $503(0.6 \%)$ of the isolates. Resistance to RHS was $2(0.4 \%)$ and to RH was 7 $(1.4 \%)$ of all tested isolates. Overall MDR-TB was observed in 12/503 (2.4\%). Of these 12 MDR-TB isolates, nine were new and three were relapse cases. Resistance to all four drugs was less than one $0.6 \%(3 / 503)$ among all tested isolates. Resistance to a single drug was higher for isoniazid $(\mathrm{H})$ $18 / 503(3.6 \%)$ of all tested isolates and 18/39 (46.2\%) of all the resistant strains. Of these 18 resistance isolates to $\mathrm{H}, 15$ were from new and 3 were from relapse cases. Resistance to other three single drugs (streptomycin, ethambutol and rifampicin) was less than $1 \%$ (Table 2). 
Table 1: Distribution of drug sensitivity pattern by sex, age group, HIV status, Health facility, smear and culture intensity and body mass index

\begin{tabular}{|c|c|c|c|c|c|}
\hline Variables & Response & Number & $\begin{array}{l}\text { Sensitive, } \\
\mathrm{N}=464(\%)\end{array}$ & $\begin{array}{l}\text { Resistance, } \\
\mathrm{N}=39(\%)\end{array}$ & P-value \\
\hline \multirow[t]{2}{*}{ Sex } & Female & 201 & $189(40.73)$ & 12 (30.77) & 0.22 \\
\hline & Male & 302 & $275(59.27)$ & $27 \quad(69.23)$ & \\
\hline \multirow[t]{5}{*}{ Age groups (yrs) } & $15-24$ & 76 & $68(14.62)$ & $8 \quad(20.51)$ & 0.62 \\
\hline & $25-34$ & 189 & $175(37.63)$ & $4(35.90)$ & \\
\hline & $35-44$ & 129 & $118(25.38)$ & 11 (28.21) & \\
\hline & $45-54$ & 68 & $64(13.76)$ & $5(12.82)$ & \\
\hline & $55+$ & 41 & $40(8.60)$ & $1(2.56)$ & \\
\hline \multirow[t]{2}{*}{ HIV status } & $\mathrm{HIV+}$ & 218 & $203(43.34)$ & $15(38.46)$ & 0.51 \\
\hline & HIV- & 285 & $261(56.66)$ & $24(61.54)$ & \\
\hline \multirow[t]{5}{*}{ Health facility } & Sekou Toure & 245 & $224(48.28)$ & $21(53.85)$ & 0.77 \\
\hline & Bugando & 87 & $80(17.24)$ & $7(17.95)$ & \\
\hline & Magu & 91 & 87 (18.75) & $4(10.26)$ & \\
\hline & Buzuruga & 45 & $41(8.84)$ & $4(10.26)$ & \\
\hline & Butimba & 35 & $32(6.90)$ & $3(7.69)$ & \\
\hline \multirow[t]{5}{*}{ Smear intensity } & Negative & 40 & $36(7.76)$ & $4(10.26)$ & 0.67 \\
\hline & 1-9 AFB & 48 & $45(9.70)$ & $3(7.69)$ & \\
\hline & $1+$ & 79 & $70(15.09)$ & $9(23.08)$ & \\
\hline & $2+$ & 97 & $90(19.4)$ & $7(17.95)$ & \\
\hline & $3+$ & 239 & $223(48.06)$ & $16(41.03)$ & \\
\hline \multirow[t]{3}{*}{ Culture intensity } & Low (1-19 colonies) & 117 & $108(23.28)$ & $9(23.08)$ & 0.45 \\
\hline & Moderate (20-100 colonies) & 70 & $62(13.36)$ & $8(20.51)$ & \\
\hline & Heavy (Confluent growth) & 316 & $294(63.36)$ & $22(56.41)$ & \\
\hline \multirow[t]{2}{*}{ Body mass index } & $<18$ & 260 & $246(53.02)$ & $14(35.90)$ & 0.04 \\
\hline & $>18$ & 243 & $218(46.98)$ & $25(64.10)$ & \\
\hline Total & & & $464(92.26)$ & $39(7.74)$ & \\
\hline
\end{tabular}

$\mathrm{AFB}=$ Acid - fast bacilli

Of those 464 isolates which were sensitive to all four drugs tested, 422 (90.95\%) were from new cases and $42(9.1 \%)$ were from relapses. Of the 39 isolates which were resistance to any anti-TB drugs, $28(71.8 \%)$ were from new smear positive, $5(12.8 \%)$ were from new smear negative and 6 $(15.4 \%)$ were from previously treated cases (relapses) (Table 3). Among the previously treated cases, $42 / 48$ (87.5\%) were sensitive to all four anti-TB drugs and only 6/48 (12.5\%) were resistant.

Table 2: Level of drug sensitivity pattern among 503 TB patients by type of drugs and form of TB

\begin{tabular}{lllllll}
\hline Drug sensitivity & \multicolumn{2}{l}{ All cases combined } & New cases & \multicolumn{3}{c}{ Relapse cases } \\
& No. & $\%$ & New & $\%$ & Relapse & $\%$ \\
\hline Sensitive to all 4 drugs (RHES) & 464 & 92.2 & & & & \\
Resistance to all 4 drugs (RHES) & 3 & 0.6 & 3 & 9.1 & 0 & 0 \\
Resistance to RHS & 2 & 0.4 & 1 & 3.0 & 1 & 16.7 \\
Resistance to HR & 7 & 1.4 & 5 & 15.2 & 2 & 33.3 \\
Resistance to H & 18 & 3.6 & 15 & 45.5 & 3 & 50.0 \\
Resistance to E & 1 & 0.2 & 1 & 3.0 & 0 & 0 \\
Resistance to R & 4 & 0.8 & 4 & 12.1 & 0 & 0 \\
Resistance to S & 4 & 0.8 & 4 & 12.1 & 0 & 0 \\
Total & 503 & $100 \%$ & 33 & 100 & 6 & 100.0 \\
\hline
\end{tabular}

$\mathrm{R}=$ Rifampicin; $\mathrm{H}$ = Isoniazid; E = Ethambutol; $\mathrm{S}=$ Streptomycin 
Table 3: Sensitivity pattern by form of TB among 503 PTB patients

\begin{tabular}{llll}
\hline TB case & Total No (\%). & Sensitive, N (\%) & Resistance, $\mathbf{N ~ ( \% ) ~}$ \\
\hline New cases & & & \\
PTB+ & $423(84.1)$ & $395(85.1)$ & $28(71.8)$ \\
PTB- & $32(6.4)$ & $27(5.8)$ & $5(12.8)$ \\
Relapse & $48(9.5)$ & $42(9.1)$ & $6(15.4)$ \\
Total & $\mathbf{5 0 3}$ & $\mathbf{4 6 4}(\mathbf{9 2 . 2})$ & $\mathbf{3 9} \mathbf{( 7 . 8 )}$ \\
\hline
\end{tabular}

PTB+ = Pulmonary sputum smear/culture positive TB; PTB- = Pulmonary sputum smear/culture negative TB

Of the 503 tested isolates, among the 40 sputum smear negative but culture positive; 30,8 and 2 were low, moderate and heavy culture intensity respectively (Table 4). Most of the heavy sputum smear intensities were also heavy culture intensity. Majority $(70 \%)$ of those with smear negative but culture positive were HIV positive (data not shown).

Table 4: Intensity of smear and culture by HIV status among 503 PTB+ patients

\begin{tabular}{lllll}
\hline Smear and culture intensities & No. & HIV+ N (\%) & HIV- N (\%) & P-value \\
\hline Smear intensity & & & & $<0.0001$ \\
$\quad$ Negative & 40 & $28(70.0)$ & $12(30.0)$ & \\
$1-9$ & 48 & $27(56.3)$ & $21(43.8)$ & \\
$1+$ & 79 & $39(49.4)$ & $40(50.6)$ & \\
$2+$ & 97 & $42(43.3)$ & $55(56.7)$ & \\
$3+$ & 239 & $82(34.3)$ & $157(65.7)$ & \\
Culture intensity & & & \\
$\quad$ Low (1-19 colonies) & 117 & $71(60.7)$ & $46(39.3)$ & \\
$\quad$ Moderate (20-100 colonies) & 70 & $27(38.9)$ & $43(61.4)$ & \\
$\quad$ Heavy (Confluent growth) & 316 & $120(38.0)$ & $196(62.0)$ & \\
\hline
\end{tabular}

PTB $+=$ Pulmonary sputum smear/culture positive TB

\section{Discussion}

Overall, our results show that the prevalence of drug resistance in the studied population is low both among new and re-treatment (relapse) cases. This implies that the Tanzanian TB and leprosy control programme is performing well in terms of patient management and also that patients adhere or comply well and most likely complete their TB treatment. None of the three isolates resistant to all four tested drugs was from relapse cases. Among the seven MDR isolates, two were relapse cases. Of the 39 cases which were resistant to any one drug or more, only $15 \%$ were from relapse cases. Although some studies have reported previous treatment to be one of the risk factors for drug resistance or poor treatment outcome (James et al., 2009); this was not the case in our study since majority of the relapse cases were not harbouring resistant strains. Interestingly, in terms of level of drug resistance, our findings though were confined to a certain geographical area, did not differ from the findings of national anti-TB drug surveillance done in the whole country in 2007 (Chonde et al., 2010).

The resistance pattern in our study did not show any association with sex, age group, health facility, previous treatment, smear or culture intensity and HIV status. Reports from other countries have shown poor treatment outcome and sex, smear positivity and association between 
TB drug resistance and HIV infection (WHO, 2008). Male sex has been associated with poor treatment outcome (James et al., 2009). Though other studies have reported association between age and drug resistance (Su et al., 1997), our study did not show any association between age and drug resistance.

Acquired rifamycin resistance has been associated with HIV infection among TB patients under treatment and anti-TB drug mal-absorption has also been documented in patient cohorts in settings of high HIV prevalence (Wells et al., 2007). However, in our study neither primary nor acquired drug resistance was associated with HIV infection. In other words, we did not find any difference in drug resistance profile among HIV positive and HIV negative TB patients. Nevertheless, the epidemiological impact of HIV on the epidemic of drug resistant TB is not well established, and may depend on several factors. For instance, at a voluntary HIV counselling and testing centre in Haiti, HIV-infected patients with TB were 3-fold more likely to have MDR-TB than were those without HIV infection (Joseph et al., 2006). According to WHO report on antituberculosis drug resistance; multi-drug resistant TB (MDR TB) has been shown to be almost twice as common in TB patients living with HIV compared to TB patients without HIV (WHO, 2008). Other studies have also reported HIV to be a risk factor for drug resistance TB (Gordin et al., 1996) and TB resistance to be more frequent among HIV-confected TB patients than those without HIV infection (Haar et al., 2007).

***The majority of HIV infected TB patients are likely to be smear negative. They tend to have lower rate of sputum smear positivity (Burman \& Jones, 2011; El-Sadr \& Tsiouris, 2003). In line with our study findings, those patients who were HIV infected had significantly low intensity of bacilli both in sputum smears and in culture compared to HIV negative TB patients. This is probably due to the fact that TB patients who are HIV-infected tend to harbour fewer bacteria due to less formation of cavities (Candice \& Joel, 2011) in the macrophage where the bacteria grow and multiply.

Our study findings show an association between drug resistance TB and BMI. To our surprise those with higher BMI $(>18 \mathrm{~kg} / \mathrm{m} 2)$ were more likely to have resistant than those with low BMI. These findings are somehow contradicting other findings in studies where low BMI has been reported to be associated with poor TB treatment outcome and normal or overweight (BMI > $25 \mathrm{Kg} / \mathrm{m}^{2}$ ) to significantly associate with lower risk of active TB than normal weight individuals (BMI, $18.5-25 \mathrm{~kg} / \mathrm{m}^{2}$ ) (James et al., 2009). Cholesterol -rich diet has also been reported to accelerate bacteriologic sterilization in pulmonary tuberculosis (Masijedi et al., 2008). From nutritional point of view, we believe that nutritional status is one of the important predictive factors of treatment outcome and BMI is one of the indicators of the nutritional and socio-economic status of the individual. The findings in our study whereby those patients with BMI $>18 \mathrm{~kg} / \mathrm{m}^{2}$ were more likely to have drug resistance are unique and need further investigation probably in a larger sample size.

Does the initial intensity of sputum smear or culture predict the sensitivity of the bacilli? In our study neither smear intensity nor culture intensity was associated with drug resistance. Nevertheless, other studies have reported that the initial high smear positivity was associated with drug resistance (James et al., 2009).

In conclusion, despite the increased number of TB cases and the high prevalence of HIV among TB patients in Tanzania, the level of anti-TB drug resistance (both initial and acquired) 
continued to remain low after over 20 years of directly observed treatment (DOTS) introduction. However, every effort should be made to maintain the status quo and to prevent the emergence of further resistance. More effort should be focused on limiting transmission of the disease by reducing the infectious cases through improved cure (particularly) of smear positive cases. Taking into consideration that rifampicin is being used throughout the six months of TB treatment; and the treatment is observed at home by a non-medical professional person, drug resistance surveillance in the country should be maintained among both new and re-treatment cases.

\section{Acknowledgements}

The authors would like to thank all the staff of the health facilities and laboratories who were involved in this study, including staff at the Mwanza Research Centre for their invaluable contribution to the success of the study. Thank to the study patients for consenting to participate in the study. The financial support of Danish International Development Assistance, via the Danish Council for Development Research and DBL-Institute for Health Research and Development (Copenhagen, Denmark) is gratefully acknowledged.

\section{Conflict of Interest}

The authors have no conflict of interest. Funding source has no influence on the reported findings.

\section{References}

Burman, W.J. \& Jones, B.E. (2003) Clinical and radiographic features of HIV-related tuberculosis. Seminars in Respiratory Infections 18, 263-271.

Bwire, R., Borgdorff, M.W., Sticht-Groh, V., Rieder, H.L., Kawuma, H.J. \& Bretzel, G. (1999) Tuberculosis chemotherapy and sputum conversion among HIV-seropositive and HIVseronegative patients in south-eastern Uganda. East African Medical Journal 76, 307-313.

Candice, K.K. \& Joel, D.E. (2011) HIV and Tuberculosis: a deadly human HIV and tuberculosis: a deadly Human. Clinical Microbiology Reviews 24, 351-376.

Chonde, T.M., Basra, D., Mfinanga, S.G., Range, N., Lwilla, F., Shirima, R.P., van Deun, A., Zignol, M., Cobelens, F.G., Egwaga, S.M. \& van Leth, F. (2010) National anti-tuberculosis drug resistance study in Tanzania. International Journal of Tuberculosis and Lung Disease 4, 967-972.

El-Sadr, W.M. \& Tsiouris, S.J. (2008) HIV-associated tuberculosis: diagnostic and treatment challenges. Seminars in Respiratory and Critical Care Medicine 29, 525-531.

Gordin, F.M., Nelson, E.T., Matts, J.P., Cohn, D.L., Ernst, J., Benator, D., Besch, C.L., Crane, L. R., Sampson, J.H., Bragg, P.S. \& El-Sadr, W. (1996) The impact of human immunodeficiency virus infection on drug-resistant tuberculosis. American Journal of Respiratory and Critical Care Medicine154, 1478-1483.

Githui, W., Kitui, F, Juma, E.S., Obwana, D., Mwai, J. \& Kwamanga, D. (1993) A comparative study on the reliability of the fluorescence microscopy and Ziehl-Neelsen method in the diagnosis of pulmonary tuberculosis. East African Medical Journal 70, 263-266. 
Haar, C.H., Cobelens, F.G., Kalisvaart, N.A., van der Have, J.J., van Gerven, P.J. \& van Soolingen, D. (2007) Tuberculosis drug resistance and HIV infection, the Netherlands. Emerging Infectious Diseases 13, 776-778.

James, C.J., Neal, C.S., Mohsen, S. \& Fitzgerald, J.M. (2009) Treatment Outcomes of Multi-drugResistant Tuberculosis: A Systematic Review and Meta-Analysis. PLoS ONE 4 (9), e6914.

Joseph, P., Severe, P., Ferdinand, S., Goh, K.S., Sola, C., Haas, D.W., Johnson, W.D., Rastogi, N., Pape, J.W. \& Fitzgerald, D.W. (2006) Multidrug-resistant tuberculosis at an HIV testing center in Haiti. Acquired Immune Deficiency Syndrome 20, 415-418.

Mahmoudi, A. \& Iseman, M.D. (1993) Pitfalls in the care of patients with tuberculosis: Common errors and their association with the acquisition of drug resistance. Journal of American Medical Association 270, 65-72.

Masjedi, M.R., Tabarsi, P., Chitsaz, E., Baghaei, P., Mirsaeidi, M., Amiri, M.V., Farnia, P., Javanmard, P., Mansouri, D. \& Velayati, A.A. (2008) Outcome of treatment of MDR-TB patients with standardized regimens, Iran, 2002-2006. International Journal of Tuberculosis and Lung Disease 12, 750-755.

Range, N., Andersen, Á.B., Magnussen, P., Mugomela, A. \& Friis, H. (2005) The effect of micronutrient supplementation on treatment outcome in patients with pulmonary tuberculosis: a randomized controlled trial in Mwanza, Tanzania. Tropical Medicine and International Health $10,826-832$.

Range, N., Magnussen, P., Mugomela, A., Malenganisho, W., Changalucha, J., Temu, M.M., Mngara, J., Krarup, H., Friis, H. \& Andersen, A.B. (2007) HIV and Parasitic Co-infections in Tuberculosis Patients: a cross-sectional study in Mwanza, Tanzania. Annals of Tropical Medicine and Parasitology 101,343-351.

Paramasivan, C.N. \& Venkataraman, P. (2004) Drug resistance in tuberculosis in India Indian Journal of Medical Research 120, 377-386.

Su, W., Lee, P., Yu, K. \& Perng, R. (1997) Drug resistance of Mycobacterium tuberculosis isolated from patients at a medical centre in Taiwan. Zhonghua Yi Xue Za Zhi (Taipei) 60, 21.

Wells, C.D., Cegielski, J.P., Lisa J.N., Kayla, F.L., Timothy, H.H., Alyssa, F., Kenneth, G.C. \& Karin, W. (2007) HIV Infection and Multi-drug Resistant Tuberculosis - The Perfect Storm. The Journal of Infectious Diseases 196, S86-S107.

WHO (2008) Anti-Tuberculosis drug resistance in the world. Report No. 4. The WHO/IUATLD Global Project on Anti-Tuberculosis drug resistance surveillance 2002-2007. WHO/HTM/TB/2008.394, World Health Organisation, Geneva 2008.

WHO (2011) Global Tuberculosis Control. WHO/HTM/TB/2011.16. World Health Organisation, Geneva. 